\title{
Structural basis for the in vitro known acyl-depsi- peptide 2 (ADEP2) inhibition to Clp 2 protease from Mycobacterium tuberculosis
}

\author{
Natasha Khandekar ${ }^{1,2}$, Snehal Singh2 ${ }^{2}$, Ruchi Shukla ${ }^{2}$, Sridevi Tirumalaraju ${ }^{3}$, Srinivas Bandaru ${ }^{4}$, \\ Tushar Banerjee ${ }^{1}$, Anuraj Nayarisseri ${ }^{*}$
}

${ }^{1}$ School of Life Science, Devi Ahilya University, Khandwa Road, Indore - 452 001, Madhya Pradesh, India; ${ }^{2 B i o i n f o r m a t i c s ~ R e s e a r c h ~}$ Laboratory, Eminent Biosciences, Vijaynagar, Indore - 452010, India; ${ }^{3}$ Mahatma Gandhi National Institute of Research \& Social Action, Hyderabad - 500029, India; "Institute of Genetics and Hospital for Genetic Diseases, Osmania University, Hyderabad - 500 016, India. Anuraj Nayarisseri - E-mail: anuraj@eminentbio.com; *Corresponding author

Received May 21, 2016; Accepted June 10, 2016; Published June 21, 2016

\begin{abstract}
:
Inhibition of Mycobacterium tuberculosis $\mathrm{Clp} 2$ protease has emerged as an attractive therapeutic option for treatment. Acyldepsipeptides (ADEPs) is known as an inhibitor for Clp 2 protease. Therefore, it is of interest to document its affinity, enzyme activity and ADME profiles. We report the predicted binding affinity of all known Clp 2 inhibitors like IDR-10001 and IDR-10011 against Clp2 protease using MolDock algorithm aided molecular docking. The predicted activity (using Molinspiration server) and ADMET properties (AdmetSAR server) were estimated for these compounds. This data suggest ADEP2 having improved binding features with Mtb Clp 2 having acceptable ADMET properties. This is in agreement with known in vitro data for ADEP2 inhibition with Mtb Clp 2 protease.
\end{abstract}

Keywords: Mycobacterium tuberculosis, Clp 2 protease, ADEPs, molecular docking, pharmacological profiling

Background:

It is estimated that each year more than 9 million new cases of tuberculosis are registered, and it kills three million people per year [1]. The WHO report shows that $3.7 \%$ patients worldwide are multi-drug resistant [2]. It is of interest to design new compounds due to total drug resistance (TDR) and multi drug resistance (MDR) [3-6]. Major energy dependent proteases are found in bacteria [7, 8]. Cutin like proteases (Clp) of Mycobacterium tuberculosis plays key role in the assembly of the mycobacterial cell wall and is essential for its growth [9]. Clps also plays a critical role in response to stress conditions [10]. The complex of Clp protease constitutes a group of dual heptameric rings structures in proteolytic subunit surrounded by a hexameric ring in ATPase subunit [11]. It is known that although $\mathrm{ClpP} 1$ and ClpP2 form tetradecamers by themselves, they are however inactive. The uncommon characteristics of this complex with both the subunits i.e. ClpP1P2 protease, makes it an atypical target for drug development [12]. The proteolysis mediated death of gram-positive bacteria (Streptococcus, Bacillus subtilis, and Enterococcus) by acyldepsipeptides (ADEPs) targeting Clps is known $[13,14]$.

In vivo and in vitro investigation have shown that synergistic binding of ADEP and ClpP promotes uncontrolled proteolytic activity directing excessive filamentation and ultimately leading to cell necrosis [15]. Therefore, it is of interest to document the structural basis of ADEPs targeting Clp2 of mycobacterium tuberculosis using modeling and binding data.

Methodology:

Selection of compounds: 
The established acyldepsipeptide compounds and its derivatives in addition to those belonging to the enopeptin class of natural product were selected from literature [16, 17]. ADEP2, ADEP3, ADEP4, IDR-10001 and IDR-10011 were selected for the binding interactions against Clp2 in the present study (Figure 1).

\section{Preparation of protein:}

The structure of Clp2 was retrieved from Protein Data Bank (PDB) with PDB ID 4U0G [18] (Figure 2). The structure was further made bonafide by preparing the protein by PrepWiz module of Schrodinger suite. The protein was first pre-processed by creating zero order bonds to metals and adding disulphide bonds and assigning the bond orders and hydrogens. Missing loops and side chains were filled using Prime Module of Schrodinger. After pre-processing, $\mathrm{H}$ bonds were assigned to the structure followed by OPLS 2005 force field energy minimization [19]. Final structure thus obtained was saved in .pdb format for further studies.

\section{Structure Similarity search:}

Similarity search was performed by Binary Finger Print Based Tanimoto similarity equation considering all the ADEPs as query molecules to retrieve compounds with similarity threshold of 95 $\%$ against NCBI's Pubchem compound database [21].

\section{Molecular docking of compounds:}

All the ligands were docked at the active site of Clp2 employing MolDock scoring function embedded in Molegro Virtual Docker (MVD) [22-24]. Docking parameters were set to maximum iteration of 1500, $0.20 \AA$ as grid resolution and maximum population size of 50. Simplex evolution was set at maximum steps of 300 with neighbourhood distance factor of 1 . Binding affinity with protein was evaluated on the basis of the internal hydrogen bonds interactions, sp2-sp2 torsions and internal ES (Internal electrostatic Interaction). Post docked energy of the ligand-receptor complex was minimized by Nelder Mead Simplex Minimization (using non-grid force field and $\mathrm{H}$ bond directionality) [25]. On the basis of re-rank score (affinity score) high affinity compound was selected from each dataset.

Table 1: The activity of the compounds and the predicted binding affinity (re-rank score) is listed.

\begin{tabular}{lcc}
\hline Compound & $\begin{array}{c}\text { Activity on } \boldsymbol{M} \text {. } \\
\text { tuberculosis+ }\end{array}$ & $\begin{array}{c}\text { Binding affinity } \\
\text { (Re-rank Score) }\end{array}$ \\
\hline ADEP2 & 25 & -142.495 \\
ADEP3 & 100 & -97.998 \\
ADEP4 & 50 & -100.089 \\
IDR-10001 & 100 & -117.216 \\
IDR-10011 & 50 & -112.300 \\
\hline
\end{tabular}

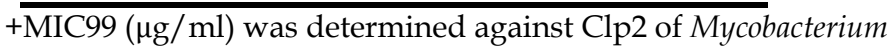
tuberculi on solid medium.

\section{Preparation of compounds:}

The ligands were optimized using LigPrep module of Schrödinger suite, 2013 (Schrodinger. LLC, New York, NY) employing OPLS 2005 force field algorithm [20]. The ionizations of the ligand were retained at the original state and were desalted and saved in sdf format.

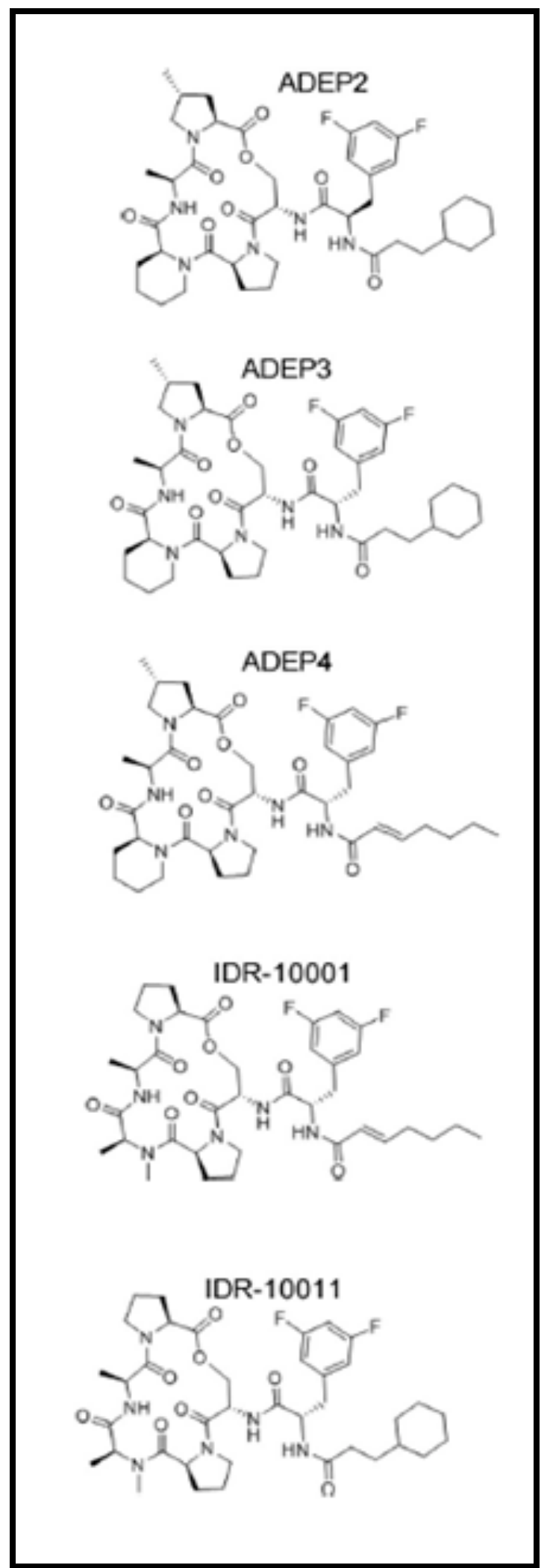

Figure 1: Structures for ADEP compounds and their derivatives. 
Bioactivity and ADMET profiling of compounds:

The complete ADMET properties were calculated using admetSAR [26]. Biological activity of the ligands was predicted using Molinspiration webserver (C) Molinspiration Cheminformatics 2014).

\section{Pharmacophore Mapping:}

Pharmacophoric mapping which involves hydrogen bond interaction, ligand interaction patterns and hydrophobic interactions were evaluated using Accelrys Discovery Studio 3.5 DS Visualizer [27].

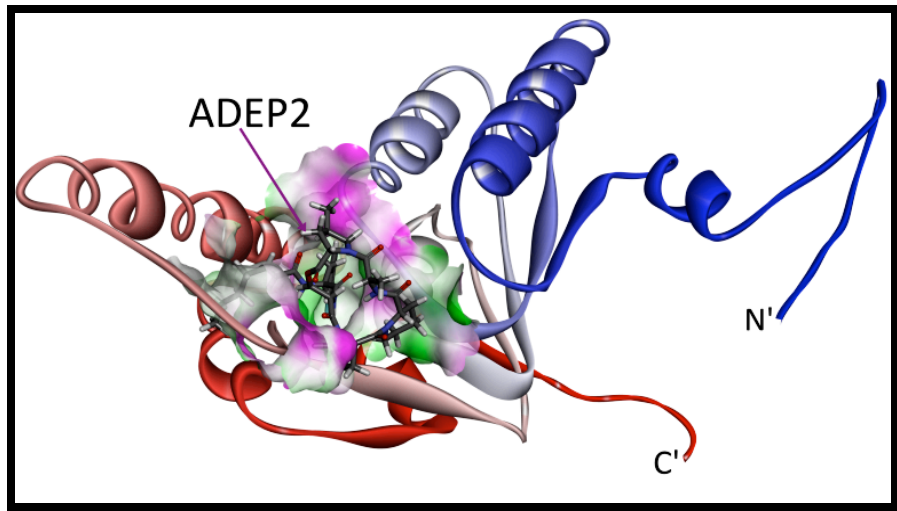

Figure 2: ADEP 2 bound at the active site of Clp 2.

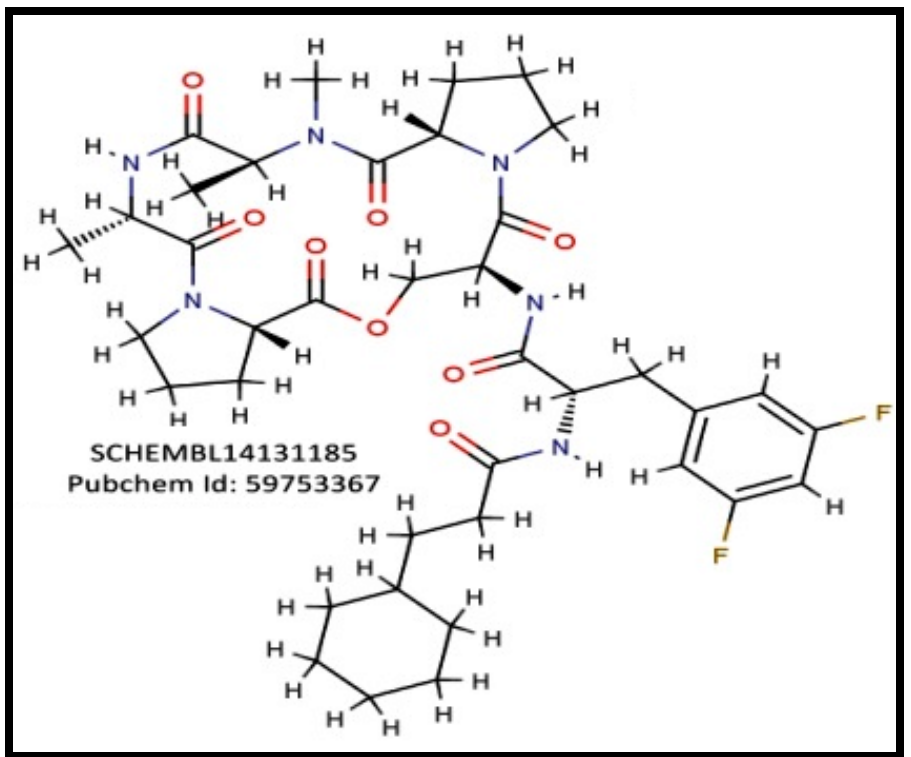

Figure 3: Structure of SCHEMBL14131185 Pubchem Id: 59753367 a similar compound to ADEP2

\section{Results and Discussion:}

Table 1 shows the re-rank (affinity) scores for Clp2 inhibitors similar to minimum inhibitory concentration (MIC) reported by Ollinger et al. (2012) [16]. Docking (re-rank) score for ADEP2 is with highest binding affinity (Table 1). Figure 2 shows ADEP2 bound at the active site of Clp2 (PDB: 4U0G). Structural analysis of ADEP2 shows that 3, 5 difluoro phenyl group connects the core structure with $\mathrm{R}$ configuration (a distinguishing feature from ADEP3 which has $S$ configuration) having superior activity $(\mathrm{MIC}=50)$ and maximum affinity (Re-rank Score $=-142.495)$. It is noted that the binding affinity results shows ADEP2 as a high affinity binder to Clp2 as described elsewhere by Ollinger et al. (2012) [16]. It shows ADEP2 to have best activity (MIC) to bring about ADEP2 mediated growth inhibition (Table 1). In addition, studies by Brötz-Oesterhelt et al. (2005) [17] also reported that ADEP2 to have an anti-bacterial activity against gram-positive bacteria in in vitro and in rodent models of bacterial infection. The binding affinity prediction that ADEP2 to be an appreciable antimicrobial agent targeting Clp2 corroborates with the data of Ollinger et al. (2012) and Brötz-Oesterhelt et al. (2005) [16, 17].

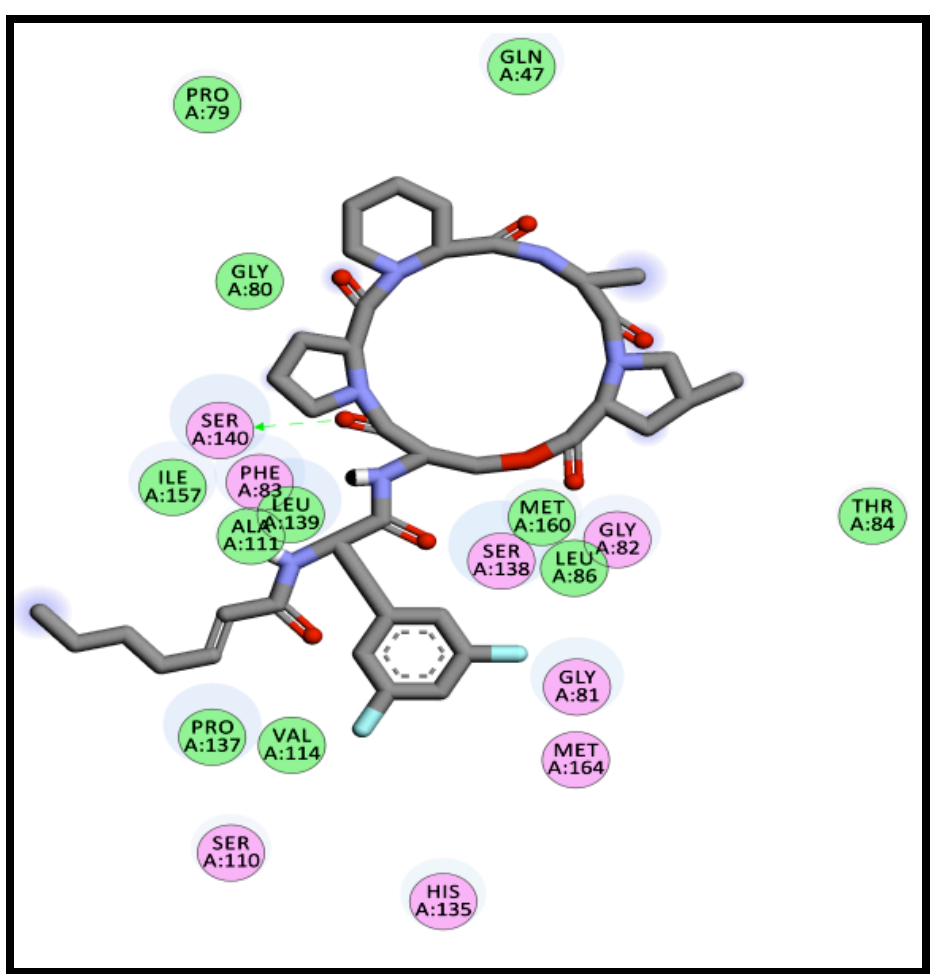

Figure 4: Ligand-receptor interactions of compound ADEP2 in the active site of Clp2 (PDB 4U0G). Residues in green contribute in van der Waals interaction while residues in pink participate in electrostatic interactions. Ser 140 form hydrogen bond acceptor from the ligand (green arrow)

Virtual screening for structures was performed against Pubchem database (taking compound ADEP2 as query) to search for better molecules with superior pharmacological profile than compound ADEP2. A total of 33 compounds structurally similar to compound ADEP2 were retrieved. All the similar compounds retrieved hitherto were docked against the Clp2 structure. Compound SCHEMBL14131185 with Pubchem Id: 59753367 (Figure 3) showed highest binding affinity amongst similar 33 compounds retrieved. It should be noted that SCHEMBL14131185 showed higher affinity to Clp2 than its parent compound ADEP2. It shows less activity for enzyme 
inhibition (Table 2) despite high predicted activity scores. The ADMET profiles are poor when compared to its parent compound ADEP2 (Table 3). In addition, compound SCHEMBL14131185 was predicted to be Ames toxic despite high good affinity.
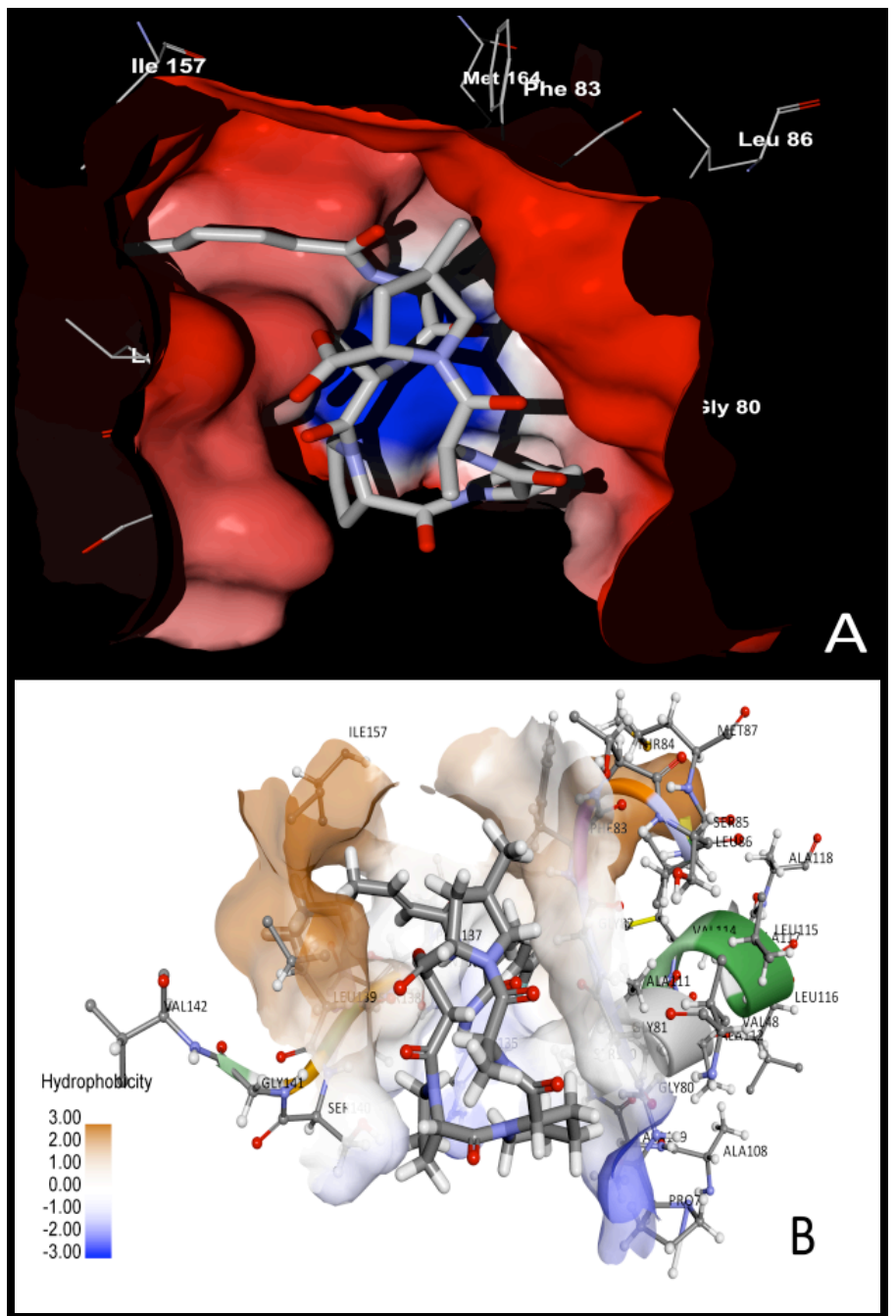

Figure 5: (A) Electrostatic Interaction of ADEP2 in Clp2. Electronegative and electropositive surface of the cavity is shown as red and blue colors respectively. White surface is electrically neutral. (B) Hydrophobic interactions of ADEP2 in the active site of receptor. Cavity is shown with hydrophobic intensities. The hydrophobic intensity of the binding site ranges from -3.00 (least hydrophobic area - blue shade) to 3.00 (highly hydrophobic area brown shade).

The appreciable binding is due to $\mathrm{H}$-bonding and electrostatic interactions for ADEP2. The descriptors energy values of external ligand interactions contribute 10.5 folds higher stability than internal ligand interactions for ADEP2. External ligand interactions were stabilized mostly by Piece wise linear potentials guided stearic energy, while the torsional strain contributes for the stability in internal ligand interactions. Table 4 shows affinity energy profile of ADEP2 and its similar SCHEMBL14131185 (Pubchem Id: 59753367).

ADEP2 shows van der Waals interactions with Pro79, Gln47, Gly80, Ile157, Leu139, Ala111. Pro137, Val114, Leu86, Met160 and Thr84 and electrostatic interactions with Ser140, Phe83, Ser138, Gly81, Gly82 and Met164. ADEP2 is a hydrogen bond donor to Ser 140 (Figure 4). The electrostatic interactions and hydrophobic interactions of ADEP2 in the binding cavity of Clp2 is show in Figure 5. These data along with the observation made by BrötzOesterhelt, et al. (2005) [17] suggests ADEp2 as a potential candidate for Clp2 inhibition.

\section{Conclusion:}

The inhibition of Mtb Clp2 by ADEP2 is known. However, its molecular basis is unknown. Therefore, it is of interest to document its binding affinity, enzyme activity and ADME profiles. The predicted activity and ADMET properties were estimated. This data suggest ADEP2 having improved binding features with Mtb Clp 2 having acceptable ADMET properties. This is in agreement with known in vitro data for ADEP2 inhibition with Mtb Clp 2 protease.

Table 2: Bioactivity prediction of Parent and similar compounds against various drug targets

\begin{tabular}{lllllll}
\hline Compound & $\begin{array}{l}\text { GPCR } \\
\text { ligand }\end{array}$ & $\begin{array}{l}\text { Ion channel } \\
\text { modulator }\end{array}$ & $\begin{array}{l}\text { Kinase } \\
\text { inhibitor }\end{array}$ & $\begin{array}{l}\text { Nuclear receptor } \\
\text { ligand }\end{array}$ & $\begin{array}{l}\text { Protease } \\
\text { inhibitor }\end{array}$ & $\begin{array}{l}\text { Enzyme } \\
\text { inhibitor }\end{array}$ \\
\hline ADEP2 & 0.15 & -0.12 & -0.36 & -0.11 & -0.17 & $1.38^{*}$ \\
ADEP3 & -0.07 & -0.30 & -0.20 & -0.30 & -0.08 & -0.18 \\
ADEP4 & -0.11 & 0.06 & -0.24 & -0.18 & -0.05 & -0.03 \\
IDR-10001 & -0.11 & -0.51 & -0.35 & -0.43 & -0.13 & -0.08 \\
IDR-10011 & -0.17 & -0.41 & -0.21 & -0.22 & -0.02 & -0.03 \\
SCHEMBL14131185 & -0.28 & -0.72 & -0.42 & 0.22 & -0.13 & -0.16 \\
\hline
\end{tabular}

* ADEP 2 showing activity highest enzyme inhibition and least activity against other drug targets testifying its target specificity against enzymes (in the present case Clp2); SCHEMBL14131185 (Pubchem Id: 59753367) 


\section{Open access}

Table 3: ADMET profiles of parent compound and its respective similar

\begin{tabular}{l|lc|lc} 
& ADEP2 & & \multicolumn{2}{l}{ SCHEMBL14131185 } \\
Pubchem Id: 59753367
\end{tabular}

${ }^{*}$ Compound SCHEMBL14131185 similar to ADEP2 demonstrating AMES toxicity, with high probability value therefore can be excluded from further pharmacological investigation

Table 4: Binding energy profile of ADEP2 and its respective similar of Clp2

\begin{tabular}{lll}
\hline Energy Descriptors & ADEP2 & SCHEMBL14131185 \\
\hline Total Energy (Rerank Score) & -142.495 & -162.543 \\
External Ligand interactions & -155.974 & -185.367 \\
Protein - Ligand interactions & -155.974 & -185.367 \\
Steric (by PLP) & -124.074 & -147.787 \\
Steric (by LJ12-6) & -27.691 & -34.671 \\
Hydrogen bonds & -4.209 & -2.91 \\
Electrostatic (short range) & -2.035 & 0 \\
Electrostatic (long range) & -0.81 & 0 \\
Internal Ligand interactions & 13.479 & 22.825 \\
Torsional strain & 7.440 & 1.88 \\
Torsional strain (sp2-sp2) & 0 & 0 \\
Hydrogen bonds & 0 & 0 \\
Steric (by PLP) & -3.882 & 4.076 \\
Steric (by LJ12-6) & 9.862 & 16.868 \\
Electrostatic & 7.673 & 0 \\
\hline SCHEMBL14131185 (Pubchem Id: 59753367$)$
\end{tabular}

References:

[1] Iademarco MF \& Castro KG. Semin Respir Infect. 2003 18: 225 [PMID: 14679472]

[2] World Health Organization. Global tuberculosis control: WHO report 2010.

[3] Davies J \& Davies D et al. Microbiol Mol Biol Rev. 2010 74: 417 [PMID: 20805405]

[4] Udwadia ZF et al. Respirology. 2012 17: 741 [PMID: 22564108]

[5] Udwadia ZF et al. J Epidemiol Community Health. 2012 67: 471 [PMID: 23155059]
[6] Babu GR \& Laxminarayan R, Tuberculosis. 2012 92: 301 [PMID: 22504008]

[7] Andrews SC et al. FEMS Microbiol Rev. 2003 27: 215 [PMID: 12829269]

[8] Lee CG et al. Annu Rev Physiol. 2011 73: 479 [PMID: 21054166]

[9] Mosser DM \& Edwards JP. Nat Rev Immunol. 2008 8: 958 [PMID: 19029990]

[10] Ollinger J et al. J Bacteriol. 2012 194: 663 [PMID: 22123255]

[11] Estorninho $\mathrm{M}$ et al. Microbiology. 2010 156: 3445 [PMID: 20688819]

[12] Ollinger J et al. J Bacteriol. 2012 194: 663 [PMID: PMC3264079]

[13] Kim YI et al. Nat Struct Biol. 2001 8: 230 [PMID: 11224567]

[14] Wawrzynow A et al. EMBO J. 1995 14: 1867 [PMID: 7743994]

[15] Sass P et al. Proc Natl Aca Sci. 2011 108: 17474 [PMID: 21969594]

[16] Ollinger J et al. J bacterial. 2012 194: 663 [PMID: 22123255]

[17] Brötz-Oesterhelt et al. Nat med. 2005 11: 1082 [PMID: 16200071]

[18] Schmitz KR et al. Proc Natl Aca Sci. 2014 111: E4587 [PMID: 25267638]

[19] Jorgensen WL \& Tirado-Rives J. Proc Natl Acad Sci U S A. 2005 102: 6665 [PMID: 15870211]

[20] Ligprep Version 2.3, (2009) Schrodinger. LLC, New York, USA

[21] Bandaru S et al. Bioinformation. 2014 10: 10 [PMID: 25489175]

[22] Bandaru S et al. Curr Top Med Chem. 2015 15: 1 [PMID: 25579570] 
[23] Sinha C et al. Curr Top Med Chem. 2015 15: 1 [PMID: 25579575]

[24] Thomsen et al. J Med Chem. 2006 49: 3315 [PMID: 16722650]

[25] Nelder JA \& Mead R. Comput J. 1965 7: 308

[26] Cheng F et al. J Chem Inf Model. 2012 52: 3099 [PMID: 23092397]

[27] Shaheen U et al. Bioinformation. 2015 11: 131 [PMID: 25914447]

Edited by P Kangueane Citation: Khandekar et al. Bioinformation 12(3): 92-97 (2016) License statement: This is an Open Access article which permits unrestricted use, distribution, and reproduction in any medium provided the original work is properly credited. This is distributed under the terms of the Creative Commons Attribution License

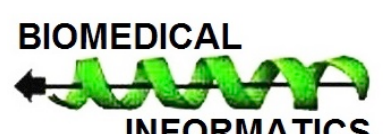

INFORMATICS 